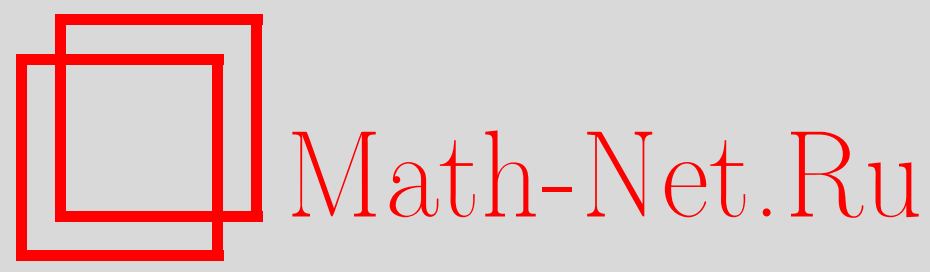

А. С. Тарасов, О сложности выпуклых стереоэдров, Maтем. заметки, 1997, том 61, выпуск 5, 797-800

DOI: https://doi.org/10.4213/mzm1563

Использование Общероссийского математического портала Math-Net.Ru подразумевает, что вы прочитали и согласны с пользовательским соглашением http://www.mathnet.ru/rus/agreement

Параметры загрузки:

IP : 35.174 .16 .151

26 апреля 2023 г., 14:25:20

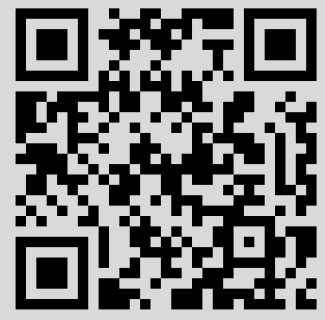




\section{О СЛОЖНОСТИ ВЫПУКЛЫХ СТЕРЕОЭДРОВ}

\section{A. C. Тарасов}

1. Введение. Счетное семейство $\mathscr{T}=\left\{P_{1}, P_{2}, P_{3}, \ldots\right\}$ замкнутых $d$-мерных многогранников назьвается разбиением $d$-мерного евклидова пространства $\mathbb{R}^{d}$, если

(Т1) многогранники покрывают пространство:

$$
\bigcup_{P_{i} \in \mathscr{T}} P_{i}=\mathbb{R}^{d} ;
$$

(Т2) никакие два многогранника не имеют общих внутренних точек:

$$
\operatorname{Int}\left(P_{i}\right) \cap \operatorname{Int}\left(P_{j}\right)=\varnothing, \quad P_{i}, P_{j} \in \mathscr{T}
$$

Мы везде будем предполагать, что многогранники $P_{i}$ ограничены и выпуклы.

Разбиение $T$ назьвается нормальным (в англоязычной литературе-стандартным), если

(Т3) любые два многогранника $P_{i}, P_{j} \in \mathscr{T}$ либо не пересекаются вовсе, либо их пересечение $P_{i} \cap P_{j}=F^{m}$ есть целая грань $F^{m}$ размерности $m, 0 \leqslant m \leqslant d-1$, общая для обоих многогранников.

В силу (Т3) для каждой $(d-1)$-грани $F^{d-1}$ многогранника $P_{i}$ в нормальном разбиении $\mathscr{T}$ найдется кроме $P_{i}$ один и только один многогранник $P_{j}$, имеюший эту же грань.

(Т4) Разбиение $\mathscr{T}$ назьвается изоәдральным, если для любых двух многогранников $P$ и $P^{\prime}$ разбиения существует симметрия разбиения $\gamma \in \operatorname{Sym}(\mathscr{T})$, переводящая $P$ в $P^{\prime}: \gamma(P)=P^{\prime}$ и $\gamma(\mathscr{T})=\mathscr{T}$.

ОпРЕДЕЛЕНИЕ. Многогранник размерности $d$, допускающий изоэдральное разбиение пространства $\mathbb{R}^{d}$, называется стереоэдром.

Стереоэдр есть ограниченньй конечньй многогранник. Для выпуклых нормальных стереоэдров имеется оценка сверху $f(h, d)$ для числа $(d-1)$-граней, зависящая только от размерности $d$ и индекса $h$ транслящионной подгрупшы $T$ в группе $\operatorname{Sym}(\mathscr{T})$ (см. [1], a также [2], [3]):

$$
f(h, d)=2\left(2^{d}-1\right)+(h-1) 2^{d} .
$$

В действительности оценка (1) справедлива не только для изоэдральных разбиений, но и для разбиений, которые распадаются на $h$ решеток выпуклых многогранников.

Так как группа симметрий $\operatorname{Sym}(\mathscr{T})$ изоэдрального разбиения - кристаллограбическая, то по теореме Шенфлиса-Бибербаха (см. [4], [5]) в $\operatorname{Sym}(\mathscr{T})$ содержится подгрупа $T$ параллельных переносов конечного индекса $h=\#(\operatorname{Sym}(\mathscr{T}) / T)$.

Если $h=1$, то $\operatorname{Sym}(\mathscr{T})=T$ есть группа параллельных переносов, и мы имеем известную оценку Минковского для числа граней параллелоэдра, которая является точной. Если $h \geqslant 1$, то оценка (1) перестает быть точной, по-видимому, для любого допустимого значения $h$. 
С другой стороны, для любой кристаллографической групшы, действующей в $\mathbb{R}^{d}$, индекс ее трансляционной подгрупшы ограничен константой $H=H(d)$, зависящей только от размерности. Таким образом, из оценки (1) следует зависящая лиш от размерности оценка сверху $f(d)$ для числа $(d-1)$-граней выпуклого стереоэдра:

$$
f(d)=2^{d}(H+1)-2 .
$$

Так как $H(2)=12$, а $H(3)=48$, то оценка Делоне (2) дает при $d=2 f(2)=50$ и при $d=3 f(3)=390$. В то же время максимальное количество сторон (плоского) стереоэдра равно 6, а количество граней у известньг на сегодня 3-мерных стереоэдров не превышает 38 (см. [6]).

Задача статьи - получить оценку (3), анонсированную в [7]. Эта оценка является улучшением оценки (2). Так согласно (3) $f(2)=44$ и $f(3)=378$.

2. Основная теорема. Пусть $T=\left\langle t_{1}, \ldots, t_{d}\right\rangle$ - трансляционная подгруппа кристаллографической групшы $\operatorname{Sym}(\mathscr{T})$.

Семейство многогранников изоэдрального разбиения распадается на $h$ смежных классов по подгруппе $T$. Заметим, что объем стереоэдра равен $V_{P}=V_{T} / h$, где $V_{T}$ - объем основного параллелепипеда решетки $T$.

ТЕорема 1. Число $(d-1)$-мерных граней выпуклого нормального $d$-мерного стереоэдра не превышает

$$
f(d)=2^{d}\left(H-\frac{1}{2}\right)-2 .
$$

ДокАЗАТЕЛЬСТво. Доказательство оценки построено следующим образом. В соответствии с тем, как группа $\operatorname{Sym}(\mathscr{T})$ разбивается на $h$ смежных классов по $T$ (смежных решеток), совокупность многогранников разбиения $\mathscr{T}$ распадается на $h$ совокупностей $\mathscr{T}_{i}$ (решеток стереоэдров). Пусть $P \in \mathscr{T}_{i}$. В [4] доказано, что число стереоэдров из той же решетки $\mathscr{T}_{i}$, имеюших с $P$ общую $(d-1)$-грань, не превышает $2\left(2^{d}-1\right)$. Далее, число стереоэдров из любой другой решетки $\mathscr{T}_{j}, j \neq i$, имеющих с $P$ общую $(d-1)$-грань, не превышает $2^{d}$. Суммируя по классам смежности, получаем оценку (1). В нашей работе оценка (1) будет снижена за счет уменьшения вклада во множество граней со стороны стереоэдров из той же решетки, что и $P$, а также из смежной решетки, получаемой из данной инверсией, при условии $h \geqslant 2^{d} d !$.

ПРЕДЛОЖЕНИЕ 1. Обгем $d$-мерного симплекса с вериинами в точках решетки $T$ кратен $V_{T} / d !($ м. $[8])$.

ПРЕДЛОЖЕНИЕ 2 [9]. Оббем выпуклой фигуры, содерэсащей $d$ отрезков, не меньше обгема симплекса, у которого все д ребер, выходящих из одной вериины, равны и параллельны данным отрезкам.

Лемма 1. Если индекс $h, h \geqslant d !$, то в разбиении $\mathscr{T}$ стереоэдр $P \in \mathscr{T}_{i}$ не может иметь более $2\left(2^{d-1}-1\right)$ общих граней со стереоэдрами из той жсе решетки $\mathscr{T}_{i}$.

ДокАЗАТЕЛЬСтво. Будем говорить, что два стереоэдра $P^{\prime}$ и $P^{\prime \prime}$ из одной решетки әквивалентны по $\bmod 2$, если $P^{\prime}=P^{\prime \prime}+\mathbf{t}$, где $\mathbf{t}-$ вектор решетки $T$, имеющий лиш четные координаты: $\mathbf{t} \equiv \mathbf{0}(\bmod 2)$. Тогда стереоэдры данной решетки $\mathscr{T}_{i}$ распадаются на $2^{d}$ классов эквивалентности по $\bmod 2$. 
В [1] было доказано, что данный стереоэдр $P \in \mathscr{T}_{i}$ в каждом классе эквивалентности решетки $\mathscr{T}_{i}$ не может иметь более 1 соседа по $(d-1)$-грани, если $P$ непринадлежит этому классу, и не может иметь ни одного соседа из того же класса эквивалентности.

Доказательство леммы основано на следующем замечании. Пусть многогранник $P$ имеет общую $(d-1)$-грань $F$ с многогранником $P_{t}=P+\mathbf{t}$, где $\mathbf{t} \in T \subseteq \operatorname{Sym}(\mathscr{T})$. Образ этой грани $F-\mathbf{t}$ является также $(d-1)$-гранью стереоэдра $P$. Возьмем точки $M \in F-\mathbf{t}$ и $M^{\prime}=M+\mathbf{t} \in F$. Мы видим, что для трансляционного вектора смежности $\mathbf{t}$ стереоэдра $P$ найдется пара точек $M, M^{\prime}$ такая, что $\overrightarrow{M M^{\prime}}=\mathbf{t}$ и $M, M^{\prime} \in P$.

Так как $P$ имеет не более двух общих $(d-1)$-граней со стереоэдрами из чужого класса эквивалентности и ни одной общей $(d-1)$-грани со стереоэдрами из своего класса эквивалентности, то стереоэдр $P$ и смежные с ним стереоэдры принадлежат попарно различным классам эквивалентности. Пусть стереоэдр $P$ имеет более $2\left(2^{d-1}-1\right)$ граней, обших со стереоэдрами из той же решетки, т.е. со стереоэдрами вида $P+T$.

В любой $(d-1)$-мерной подрешетке решетки $T$ лежит не более $2^{d-1}$ классов эквивалентности mod2. Поэтому множество всех трансляционных векторов смежности $\mathbf{t}_{i}$ не может лежать в одном $(d-1)$-мерном подпространстве и, следовательно, векторы $\mathbf{t}_{i}=\overrightarrow{M_{i} M_{i}^{\prime}}$ линейно независимы, где $M_{i}, M_{i}^{\prime} \in P$. Поэтому для объема справедлива оценка $V_{P}>V\left(\operatorname{Conv}\left(\left\{\bigcup_{i}\left[M_{i}, M_{i}^{\prime}\right]\right\}\right)\right) \neq 0$. В силу предложения 2 объем $V\left(\operatorname{Conv}\left(\left\{\bigcup_{i}\left[M_{i}, M_{i}^{\prime}\right]\right\}\right)\right)$ не меньше объема $V_{s}$ невырожденного симплекса $S$, натянутого на $d$ линейно независимых векторов $\mathbf{t}_{i}, i=1,2, \ldots, d$. Так как $\mathbf{t}_{i} \in T$, то $V_{s} \geqslant V_{T} / d !$, где $V_{T}$ - объем фундаментального параллелепипеда решетки $T$.

Так как $h \geqslant d$ !, то

$$
V_{P}>V\left(\operatorname{Conv}\left(\left\{\bigcup_{i}\left[M_{i}, M_{i}^{\prime}\right]\right\}\right)\right) \geqslant \frac{1}{d !} V_{T} \geqslant \frac{1}{h} V_{T},
$$

что противоречит соотношению $V_{P}=V_{T} / h$. Лемма 1 доказана.

Допустим, что в группе $\operatorname{Sym}(\mathscr{T})$ имеется движение $\gamma$, которое есть суперпозиция некоторой инверсии в точке и трансляции (каждое из них в отдельности может не принадлежать групе $\operatorname{Sym}(\mathscr{T}))$. C другой стороны, такая суперпозиция $\gamma$ может быть представлена в виде чистой инверсии (вообще говоря, в другой точке).

Лемма 2. Пусть индекс $h, h \geqslant 2^{d} d$ !, и группа $\operatorname{Sym}(\mathscr{T})$ содержит инверсию $\gamma$. Обозначим через $I(P)$ мнохсество всех стереоэдров из решетки $\gamma(P)+T$, которые имеют с $Р$ общую $(d-1)$-грань. Тогда \# $(I(P)) \leqslant 2^{d-1}$.

ДокАЗАТЕЛЬСтво. Пусть точка $O$ - центр тяжести многогранника $P$, которая в силу вьпуклости $P$ есть внутренняя точка многогранника $P$ с центром $O$.

Пусть $P_{i}$ - стереоэдр из $I(P)$, смежньй с $P$ по общей $(d-1)$-грани $F_{i}$, и пусть $\gamma_{i} \in$ $\operatorname{Sym}(\mathscr{T})$ такое, что $\gamma_{i}(P)=P_{i}$. Инволюция $\gamma_{i}$ переводит грань $F_{i}$ в себя. Следовательно, единственная неподвижная точка $M_{i}$ инверсии $\gamma_{i}$ является внутренней точкой грани $F_{i}$. Заметим, что $M_{i}$ - середина отрезка $O O_{i}$, а вектор $\overrightarrow{O_{i} O_{j}}-$ примитивньй вектор решетки $T$, т.е. $\overrightarrow{O_{i} O_{j}} \not \equiv 0(\bmod 2)$ (доказательство см. в [1]-[3]).

Предположим, что $P$ имеет больше $2^{d-1}$ граней, обших со стереоэдрами из решетки $I(P)$, и, следовательно, столько же точек $O_{i}$. Отсюда, так как центры $O_{i}$ принадлежат попарно различным классам эквивалентности по $\bmod 2$, то множество всех центров $O_{i}$ не может лежать ни в каком $(d-1)$-мерном подпространстве. Поэтому объем 
$V\left(\operatorname{Conv}\left(\left\{O_{i}\right\}\right)\right) \neq 0$ и, следовательно,

$$
V\left(\operatorname{Conv}\left(\left\{O_{i}\right\}\right)\right)=\frac{k}{d !} V_{T} \geqslant \frac{1}{d !} V_{T} .
$$

С другой стороны, $P \supset \operatorname{Conv}\left(\left\{M_{i}\right\}\right)$ и

$$
V(P)>V\left(\operatorname{Conv}\left(\left\{M_{i}\right\}\right)\right)=\frac{1}{2^{d}} V\left(\operatorname{Conv}\left(\left\{O_{i}\right\}\right)\right) \geqslant \frac{1}{2^{d}} \cdot \frac{1}{d !} \cdot V_{T} \geqslant \frac{1}{h} V_{T},
$$

что противоречит равенству $V_{P}=V_{T} / h$. Лемма доказана.

Чтобы получить теорему, заметим, что для кристаллографической групшы $\operatorname{Sym}(\mathscr{T})$ с максимальным индексом $H$ транслящионной подгрупш $T$ верны следующие утверждения:

1) $\operatorname{Sym}(\mathscr{T})$ содержит инверсию $\gamma$;

2) $H \geqslant 2^{d} n$ !.

Это действительно так, потому что инверсия присутствует как элемент симметрии в любой решетке, а индекс полной группы кубической решетки, которая при $d>3$ имеет, вообще говоря, не максимальньй индекс, равен $h=2^{d} n$ ! .

Теорема доказана.

Московский государственный университет

Поступило

им. М.В. Ломоносова

\section{СПИСОК ЦИТИРОВАННОЙ ЛИТЕРАТУРЫ}

1. Делоне Б. Н., Сандакова Н. Н. // Изв. АН СССР. Сер. матем. 1961. Т. 64. С. $28-51$. 2. Штогрин М. И. Правильные разбиения Дирихле-Вороного для второй триклинной группы. Тр. МИАН. Т. 123, 1973. 3. Dolbilin N., Dress A., Huson D. Two finiteness theorems for periodic tilings of $d$-dimensional Euclidean space // Discrete Comput. Geom. (to appear). 4. Schoenflies A. Kristallsysteme und Kristallstruktur. Leipzig, 1891. 5. Bieberbach Z. // Math. Ann. 1911. V. 70. P. 297-336. 6. Engel P. Geometric Crystallography. Dordrecht, 1986. 7. Tarasov A. A new upperbound for the number of $(d-1)$-faces of a convex $d$-stereohedron // Abstracts of the 3rd Geometry Festival. Budapest, 1996. Р. 17. 8. Касселс Д. Введение в геометрию чисел. М.: Мир, 1969. 9. McMullen P. // Math. Proc. Cambridge Philos. Soc. 1982. V. 91. P. 91-97. 